\title{
Investigation of plasma response influence on the penetration of an external low frequency helical perturbation into a tokamak edge plasma
}

\author{
I.M. Pankratov, A.Ya. Omelchenko, V.V. Olshansky and \\ K.H. Finken ${ }^{1}$ \\ Institute of Plasma Physics National Science Center 'Kharkov Institute of Physics and \\ Technology', Akademicheskaya str., 1, 61108 Kharkov, Ukraine \\ ${ }^{1}$ Institut fuer Plasmaphysik, Forschungszentrum Juelich GmbH, Association Euroatom-FZJ, \\ Trilateral Euregio Cluster, D-52425 Juelich, Germany \\ E-mail: pankratov@ipp.kharkov.ua
}

Received 29 October 2003, in final form 16 April 2004

Published 11 May 2004

Online at stacks.iop.org/NF/44/S37

DOI: $10.1088 / 0029-5515 / 44 / 6 / \mathrm{S} 04$

\begin{abstract}
The plasma response influence on the penetration of an external low frequency helical perturbation into a tokamak edge plasma has been studied on the basis of the two-fluid MHD equations in the linear approximation for the cylindrical model. Components of the penetrating field, the plasma flows, the perturbation current density distribution and the radial profile of the poloidal driven force density are found in the resonant region and investigated for the parameters of the TEXTOR-DED, CSTN-IV and HYBTOK-II tokamaks. Preliminary comparisons with the CSTN-IV and HYBTOK-II experiments are made and a good qualitative agreement has been found.
\end{abstract}

PACS numbers: 52.55.Dy, 52.55.Fa, 52.55.Rk

\section{Introduction}

The control of plasma edge behaviour is the main purpose of the Dynamic Ergodic Divertor (DED) Project of TEXTOR [1]. The DED helical coils create a specific topology of the magnetic field at the plasma edge, where external rotating DED helical perturbations with poloidal number $m$ and toroidal number $l$ are resonant on the magnetic surfaces $q\left(r_{\text {res }}\right)=m / l$ ( $q(r)$ is the safety factor). This topology must be investigated with the plasma response taken into account.

In this paper the general solutions of the two-fluid resistive MHD equations (components of the penetrating field, the perturbation current density distribution and the radial profile of the poloidal driven force density) are found and investigated in the cylindrical approximation. A detailed investigation of plasma response is carried out in the resonant region. The poloidal plasma rotation due to an equilibrium radial electric field, the toroidal plasma rotation with homogeneous velocity and the ion diamagnetic drift are taken into account. The approaches of high parallel electron heat conductivity and incompressible plasma motion are used. For the cases considered in this paper the resistive effects dominate in a broader region than that defined by the Alfvén resonances (compare with [2]).

The radial profiles of the radial and poloidal magnetic components of a penetrating external rotating helical field were measured using a small magnetic probe in the plasma of CSTN-IV [3, 4] and HYBTOK-II [5, 6] tokamaks. Preliminary comparisons of our calculated profiles with these measured profiles show a good qualitative agreement.

This paper is organized as follows. The basic equations are derived in section 2 . In sections 3 and 4 the solutions of these equations are presented for CSTN-IV, HYBTOK-II and TEXTOR-DED. Conclusions are given in section 5.

\section{Basic equations}

We start from the two-fluid MHD equations for continuity, momentum and energy conservation for plasma ions and electrons and the Maxwell's equations. The electron-ion collision frequency is higher than the frequency of the external perturbation. The electron inertia and electron stress tensor are neglected. Then, as usual way [7], we consider the plasma 
equation of motion

$$
\rho\left(\frac{\partial \boldsymbol{V}}{\partial t}+(\boldsymbol{V} \cdot \nabla) \boldsymbol{V}\right)=-\nabla p-\nabla \cdot \boldsymbol{\pi}_{i}+[\boldsymbol{J} \times \boldsymbol{B}]
$$

and the generalized Ohm's Law

$$
\begin{aligned}
\frac{\boldsymbol{J}_{\|}}{\sigma_{\|}}+\frac{\boldsymbol{J}_{\perp}}{\sigma_{\perp}}=\boldsymbol{E} & +[\boldsymbol{V} \times \boldsymbol{B}]+\frac{\nabla p_{\mathrm{e}}}{e n}-\frac{[\boldsymbol{J} \times \boldsymbol{B}]}{e n} \\
& +\left(\frac{0.71}{e}\right) \nabla_{\|} T_{\mathrm{e}}, \quad(e>0),
\end{aligned}
$$

where $n$ and $\rho$ are the plasma and plasma mass densities, $p=p_{\mathrm{e}}+p_{\mathrm{i}}$ is the total pressure, $\boldsymbol{J}$ the total current density, $\pi_{i}$ the ion gyroviscosity tensor, and $\sigma_{\|}$and $\sigma_{\perp}$ are the parallel and perpendicular (with respect to the magnetic field $\boldsymbol{B}$ ) conductivities, respectively. Earlier equations (1) and (2) were used for the stability analysis of the reconnecting mode [8]. The parallel electron heat-conductivity coefficient is assumed to be large. Hence, in our approximation $\left(T_{\mathrm{e}}\right.$ is the electron temperature)

$$
\boldsymbol{B} \cdot \nabla T_{\mathrm{e}}=0 .
$$

We consider a current carrying cylindrical plasma whose axis is taken as the $z$-direction. The external axial magnetic field $B_{z 0}$ is large with respect to the poloidal magnetic field $B_{\theta 0}$ produced by the axial current $J_{z 0}$. The perturbation values depend on the azimuthal angle $\theta$, the coordinate $z$ and the time $t$ as $\exp [\mathrm{i}(m \theta-k z-\omega t)]$, where $k=l / R, R$ plays the role of the tokamak major radius and $\omega$ is the frequency of the external perturbation. We use the approximation of an incompressible plasma motion $\nabla \cdot \boldsymbol{V}^{\sim}=0$, but the incompressibility for electrons is not assumed $\left(\nabla \cdot \boldsymbol{V}_{e}^{\sim} \neq 0\right)$.

We follow a procedure outlined in this paper [8]. After applying the operator $\nabla \times(\cdots)$ to the linearized equation (1), for the $z$-component we obtain the first equation for perturbations of radial components of the plasma velocity $V_{r}^{\sim}$ and magnetic field $B_{r}^{\sim}$

$$
\begin{aligned}
& \frac{\mathrm{d}}{\mathrm{d} r}\left(r \omega^{\prime} \rho \frac{\mathrm{d}}{\mathrm{d} r}\left(r V_{r}^{\sim}\right)\right)-m^{2} \omega^{\prime} \rho V_{r}^{\sim} \\
& \quad=-\frac{F(r)}{\mu_{0}}\left[\frac{\mathrm{d}}{\mathrm{d} r}\left(r \frac{\mathrm{d}}{\mathrm{d} r}\left(r B_{r}^{\sim}\right)\right)-m^{2} B_{r}^{\sim}\right]+m \frac{\mathrm{d} J_{z 0}}{\mathrm{~d} r}\left(r B_{r}^{\sim}\right)
\end{aligned}
$$

where

$$
\begin{aligned}
& \omega^{\prime}=\omega+\left(\frac{m}{r}\right)\left(\frac{E_{r 0}}{B_{z 0}}\right)+k V_{z 0}, \\
& F(r)=\boldsymbol{k} \cdot \boldsymbol{B}_{0}=\frac{m}{r} B_{\theta 0}-k B_{z 0} .
\end{aligned}
$$

From Ampere's Law

$$
\frac{1}{r} \frac{\mathrm{d}}{\mathrm{d} r}\left(r B_{\theta 0}\right)=\mu_{0} J_{z 0}
$$

and using equation (5) for $F(r)$ ( $B_{z 0}$ is constant), we find that

$$
\frac{\mathrm{d} J_{z 0}}{\mathrm{~d} r}=\left(\frac{1}{\mu_{0} m}\right)\left(\frac{r \mathrm{~d}^{2} F}{\mathrm{~d} r^{2}}+3 \frac{\mathrm{d} F}{\mathrm{~d} r}\right) .
$$

From the radial component of Faraday's Law

$$
\nabla \times \boldsymbol{E}^{\sim}=\mathrm{i} \omega \boldsymbol{B}^{\sim},
$$

$\left(\boldsymbol{E}^{\sim}\right.$ and $\boldsymbol{B}^{\sim}$ are perturbations of the electric and magnetic fields), using the linearized form of equation (2), we find the second equation for $V_{r}^{\sim}$ and $B_{r}^{\sim}$

$$
\begin{aligned}
& -\mathrm{i} \omega_{i} B_{r}^{\sim}=\mathrm{i} F(r) V_{r}^{\sim} \\
& +\frac{1}{\mu_{0} \sigma_{\|}} \frac{1}{r}\left[\frac{1}{r} \frac{\mathrm{d}}{\mathrm{d} r} r \frac{\mathrm{d}}{\mathrm{d} r}\left(r B_{r}^{\sim}\right)-\frac{m^{2}}{r^{2}}\left(r B_{r}^{\sim}\right)\right],
\end{aligned}
$$

where

$$
\omega_{i}=\omega^{\prime}-\left(\frac{m}{r}\right)\left(\frac{1}{e n_{0} B_{z 0}}\right)\left(\frac{\mathrm{d} p_{i 0}}{\mathrm{~d} r}\right) .
$$

In equations (4) and (7) the terms $k V_{z}^{\sim}$ and $k B_{z}^{\sim}$ are neglected $(k r<m)$. Here, we included the poloidal plasma rotation due to the existence of an equilibrium radial electric field $E_{r 0}$, the toroidal plasma rotation with homogeneous velocity $V_{z 0}$ and also took into account the ion diamagnetic drift. We recall that the ion gyroviscosity tensor $\boldsymbol{\pi}_{i}$ compensates the drift diamagnetic effect in equation (4) (see, e.g. $[8,9])$. It is assumed that the equilibrium quantities are slowly varying.

After some algebra, from equations (4) and (7) we obtain the closed system for $V_{r}^{\sim}$ and $B_{r}^{\sim}$ :

$$
\begin{aligned}
& \frac{\mathrm{d}}{\mathrm{d} r} r \omega^{\prime} \rho \frac{\mathrm{d}}{\mathrm{d} r}\left(r V_{r}^{\sim}\right)-\left(\omega^{\prime} m^{2} \rho+\mathrm{i} \frac{r^{2}}{\delta^{2}} \frac{F^{2}(r)}{\mu_{0} \omega}\right) V_{r}^{\sim} \\
& \quad=\left(\mathrm{i} \frac{r^{2}}{\delta^{2}} \frac{\omega_{i}}{\omega} F(r)+r^{2} \frac{\mathrm{d}^{2} F}{\mathrm{~d} r^{2}}+3 r \frac{\mathrm{d} F}{\mathrm{~d} r}\right) \frac{B_{r}^{\sim}}{\mu_{0}}, \\
& \frac{\mathrm{d}}{\mathrm{d} r} r \frac{\mathrm{d}}{\mathrm{d} r}\left(r B_{r}^{\sim}\right)-\left(m^{2}-\mathrm{i} \frac{r^{2}}{\delta^{2}} \frac{\omega_{i}}{\omega}\right) B_{r}^{\sim}=-\mathrm{i} \frac{r^{2}}{\delta^{2}} \frac{F(r) V_{r}^{\sim}}{\omega},
\end{aligned}
$$

where

$$
\delta=\frac{1}{\sqrt{\mu_{0} \sigma_{\|} \omega}}
$$

The value $F(r)$ is equal to zero inside the plasma, $F\left(r_{\text {res }}\right)=0$, when $q\left(r_{\text {res }}\right)=m / l\left(q(r)=r B_{z 0} / R B_{\theta 0}\right)$. The region near $r \approx r_{\text {res }}$ is the resonant (interaction) zone. Outside the resonant zone the terms proportional to $1 / \delta^{2}$ become large in equations (9) and (10). Hence, outside the resonant zone the matching condition takes the form

$$
V_{r}^{\sim}(r) \approx-\frac{\omega_{i} B_{r}^{\sim}(r)}{F(r)}
$$

Recall that an expression like equation (12) is well known in the ideal MHD (see, e.g. [10]) and it was used as a matching condition also in this paper [8]. Note that because $\left|B_{r}^{\sim}\right|$ grows towards the antenna, $\left|V_{r}^{\sim}\right|$ has a finite value when $r \rightarrow a$.

Unlike the basic equations of the paper [11] (the one-fluid MHD) the equations (9) and (10) contain two frequencies: $\omega^{\prime}$ and $\omega_{i}$. Sometimes the two-fluid MHD model is used under assumptions that both ion and electron responses to the perturbations are incompressible and the ion and electron thermal conductivities are low enough (see [8] and review paper [12]). In the frame of such a model the investigation was made in the report [13]. However in our opinion, the model considered in this paper (the approximation of high parallel electron heat conductivity, see also [9]) corresponds more to experimental conditions. 
To understand the Alfvén resonances that may occur, $V_{r}^{\sim}$ from equation (10) may be substituted into equation (9) to obtain the single fourth-order equation:

$$
\begin{aligned}
\mathrm{i} \omega \delta^{2} & \left(\frac{1}{r} \frac{\mathrm{d}}{\mathrm{d} r} r \omega^{\prime} \rho \frac{\mathrm{d}}{\mathrm{d} r}-\omega^{\prime} \rho \frac{m^{2}}{r^{2}}\right) \\
& \times\left[\frac{1}{F(r)}\left(\frac{1}{r} \frac{\mathrm{d}}{\mathrm{d} r} r \frac{\mathrm{d}}{\mathrm{d} r}-\frac{m^{2}}{r^{2}}\right)\left(r B_{r}^{\sim}\right)\right] \\
& +\frac{\rho}{F(r)}\left(\frac{F^{2}(r)}{\mu_{0} \rho}-\omega_{i} \omega^{\prime}\right)\left[\frac{1}{r} \frac{\mathrm{d}}{\mathrm{d} r} r \frac{\mathrm{d}}{\mathrm{d} r}-\frac{m^{2}}{r^{2}}\right]\left(r B_{r}^{\sim}\right) \\
& -\frac{\mathrm{d}}{\mathrm{d} r}\left(\omega^{\prime} \rho\right) \cdot \frac{\mathrm{d}}{\mathrm{d} r}\left(\frac{\omega_{i}}{F(r)}\left(r B_{r}^{\sim}\right)\right) \\
& -\omega^{\prime} \rho\left(\frac{1}{r} \frac{\mathrm{d}}{\mathrm{d} r}\left[r \frac{\mathrm{d}}{\mathrm{d} r}\left(\frac{\omega_{i}}{F(r)}\right) \cdot\left(r B_{r}^{\sim}\right)\right]\right. \\
& \left.+\frac{\mathrm{d}}{\mathrm{d} r}\left(\frac{\omega_{i}}{F(r)}\right) \cdot \frac{\mathrm{d}}{\mathrm{d} r}\left(r B_{r}^{\sim}\right)\right) \\
& -\frac{1}{r \mu_{0}}\left(r \frac{\mathrm{d}^{2} F}{\mathrm{~d} r^{2}}+3 \frac{\mathrm{d} F}{\mathrm{~d} r}\right) \cdot\left(r B_{r}^{\sim}\right)=0 .
\end{aligned}
$$

When the first term in equation (13) is small, this equation leads to the appearance of two Alfvén resonance layers outside the interaction zone $F\left(r_{\text {res }}\right)=0$, if $\omega_{i} \omega^{\prime}>0$. The locations of these resonances are determined by the $\omega^{\prime}$ and $\omega_{i}$ frequencies (compare with [14]).

$$
\frac{\Delta r_{A}}{r_{\mathrm{res}}}= \pm \frac{\sqrt{\omega_{i} \omega^{\prime}}}{\omega Q}
$$

Here, $Q=l S V_{z A} / \omega R, V_{z A}=B_{z 0} / \sqrt{\mu_{0} \rho}, S=\left(r q^{\prime} / q\right)_{r=r_{\mathrm{res}}}$. In the opposite case, the resistive effects dominate in a broader region than that defined by the Alfvén resonances. From equation (13) the following estimate of the half width of the interaction (resonant) zone can be made (see also [15]):

$$
\Delta r \sim\left(\frac{2 r_{\text {res }} \delta}{Q}\right)^{1 / 2} \frac{\left|\omega^{\prime}\right|^{1 / 4}}{\omega^{1 / 4}} .
$$

When the Doppler shifted frequency decreases (increases), the width of the interaction zone also decreases (increases).

The Alfvén resonances occur if

$$
\left|\omega_{i} \| \omega^{\prime}\right|^{0.5}>\left(\frac{2 \delta Q}{r_{\text {res }}}\right) \omega^{1.5}
$$

Equations (9) and (10) were solved without the assumption that the vacuum perturbation of the magnetic field plays a dominant role and the plasma response is small. The projection collocation method with the Hermite basis functions of third order was applied for the numerical solution of equations (9) and (10) [16]. Equation (12) was used as the boundary condition for $V_{r}^{\sim}$. The regularity condition for $B_{r}^{\sim}$ was used at $r=0$, and at $r=a$ the boundary condition for $B_{r}^{\sim}$ was found by using the analytical solution from [11].

Below, we neglect the ion diamagnetic drift and set $\omega_{i}=$ $\omega^{\prime}, \omega^{\prime}=2 \pi f^{\prime}$. In figures 1-3 the solutions of equations (9) and (10) $B_{r, \theta}^{\sim}$ and $V_{r, \theta}^{\sim}$ are normalized to the values $B_{r}^{\mathrm{vac}}\left(r_{\mathrm{res}}\right)$ and $V_{r A}=B_{r}^{\text {vac }} /\left.\sqrt{\mu_{0} \rho}\right|_{r=r_{\text {res }}}$, respectively.
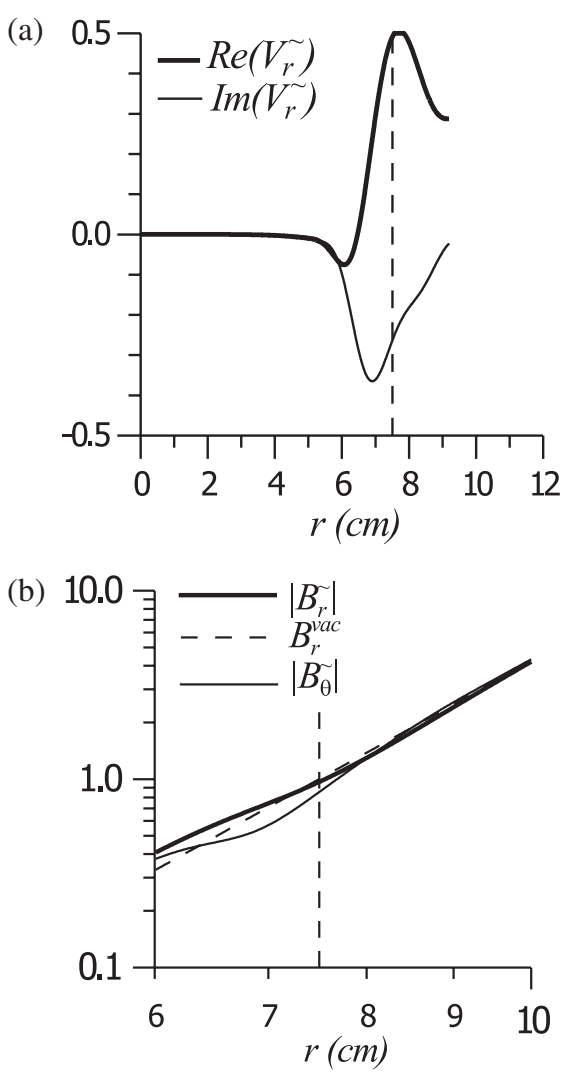

Figure 1. The CSTN-IV tokamak: (a) the radial profile of the velocity $V_{r}^{\sim} ;(b)$ profiles of the magnetic perturbation amplitudes $\left|B_{r}^{\sim}\right|,\left|B_{\theta}^{\sim}\right|$.

\section{Tokamaks CSTN-IV and HYBTOK-II}

In figure 1 the calculations for the CSTN-IV experiments $[3,4]$ are presented for the case $f^{\prime}=20 \mathrm{kHz}(R=0.4 \mathrm{~m}$, $a=0.1 \mathrm{~m}, r_{\text {res }}=7.5 \mathrm{~cm}, m=6, l=1, B_{z 0}=0.086 \mathrm{~T}$, $\left.n_{\mathrm{pl}}=1.5 \times 10^{18} \mathrm{~m}^{-3}\right)$. For $f=20 \mathrm{kHz}$ we take the skin depth value $\delta=2 \mathrm{~cm}\left(T_{\mathrm{e}} \approx 10 \mathrm{eV}\right)$. In these experiments it was found that $\left|B_{r}^{\sim}\right|$ was amplified almost over the whole radial range by up to a few ten per cent of the vacuum field, while $\left|B_{\theta}^{\sim}\right|$ was attenuated at around the resonant surface and amplified at $r<6.5 \mathrm{~cm}$. Our calculations show that in these experiments a very wide interaction region occurred (figure $1(a)$ ) and the plasma response was not so strong (see also [11]).

Note, that in the experiments $[4,6]$ for the case of offresonance to the helicity of perturbation, the modification in $\left|B_{r}^{\sim}\right|$ and $\left|B_{\theta}^{\sim}\right|$ (compared with the vacuum field) was not observed for $r>6 \mathrm{~cm}$ in the resonant zone near $q\left(r_{\text {res }}\right)=6$. But for $r<6 \mathrm{~cm}$ an amplification of $\left|B_{r}^{\sim}\right|$ and $\left|B_{\theta}^{\sim}\right|$ was obtained that was not a result of resonant interaction of the external perturbation with plasma at $r_{\text {res }}=7.5 \mathrm{~cm}$.

In the case of the resonant helical perturbation (at $r_{\text {res }}=7.5 \mathrm{~cm}$ ) the calculated profiles of $\left|B_{r}^{\sim}\right|$ and $\left|B_{\theta}^{\sim}\right|$ qualitatively confirm the experimentally measured profiles: $\left|B_{r}^{\sim}\right|$ amplification of the order of $30 \%$ of the vacuum field is found for $6 \mathrm{~cm}<r<7.5 \mathrm{~cm}$ (figure $1(b)$ ). The attenuation of $\left|B_{\theta}^{\sim}\right|$ around $r \approx r_{\text {res }}$ and then the $\left|B_{\theta}^{\sim}\right|$ amplification are observed at $r<6.3 \mathrm{~cm}$.

In figure 2 the calculations for the HYBTOK-II experiments $[5,6]$ are presented $(R=0.4 \mathrm{~m}, a=0.11 \mathrm{~m}$, 

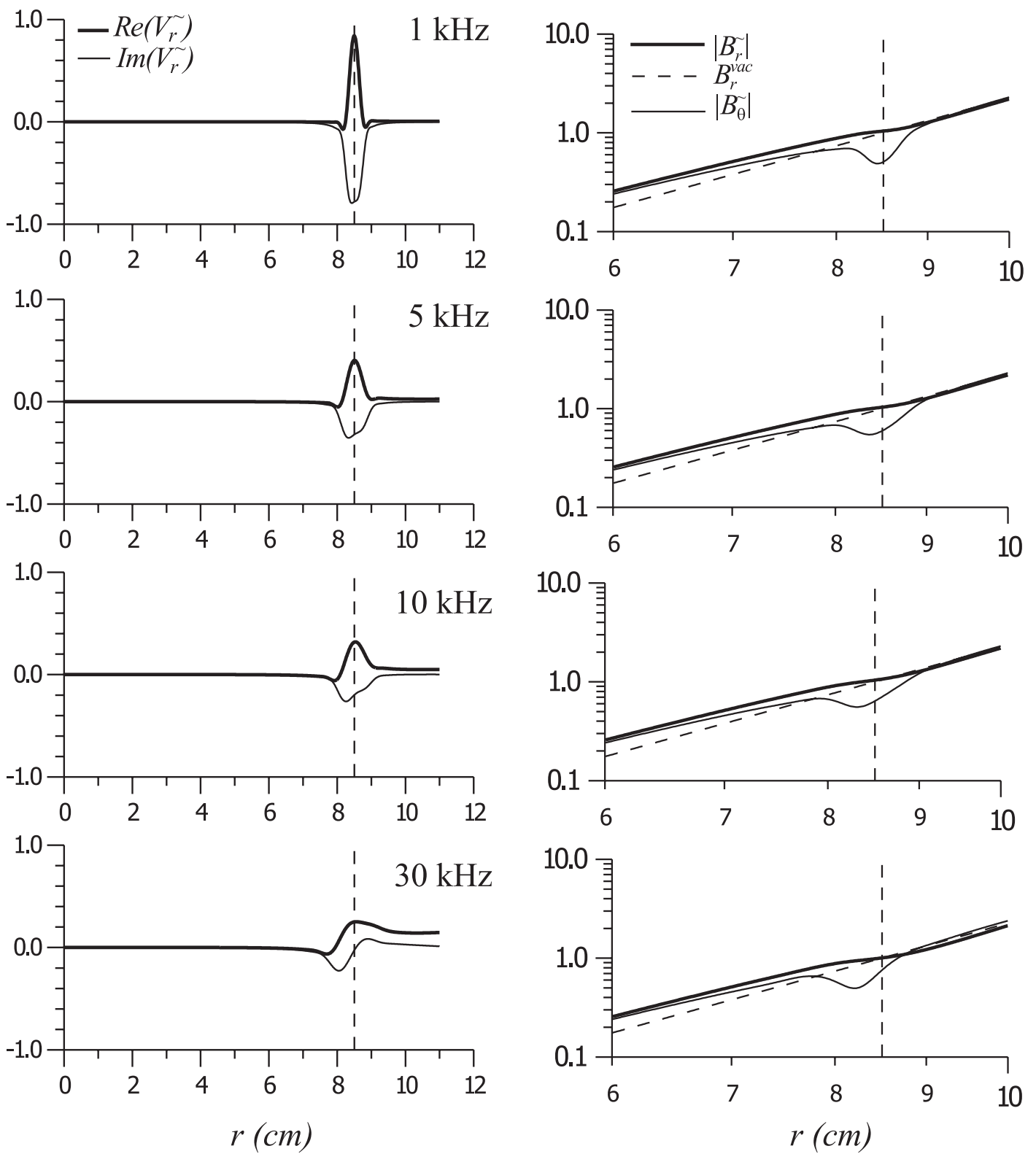

Figure 2. The HYBTOK-II tokamak radial profiles of the velocity $V_{r}^{\sim}$ and profiles of the magnetic perturbation amplitudes $\left|B_{r}^{\sim}\right|,\left|B_{\theta}^{\sim}\right|$ for the various frequency values.

$r_{\text {res }}=8.5 \mathrm{~cm}, m=6, l=1, B_{z 0}=0.27 \mathrm{~T}, n=$ $\left.1.5 \times 10^{18} \mathrm{~m}^{-3}\right)$. Four cases are considered: $f^{\prime}=1 \mathrm{kHz}$, $f^{\prime}=5 \mathrm{kHz}, f^{\prime}=10 \mathrm{kHz}$ and $f^{\prime}=30 \mathrm{kHz}$. For $f=30 \mathrm{kHz}$ we take the skin depth value $\delta=1 \mathrm{~cm}\left(T_{\mathrm{e}} \approx 20 \mathrm{eV}\right)$. In our calculations a stronger plasma response is observed in comparison with the CSTN-IV tokamak calculated data: the stronger amplification of $\left|B_{r}^{\sim}\right|$ and $\left|B_{\theta}^{\sim}\right|$ is found towards the plasma depth. The resonant nature of the interaction is clearly visible in figure 2 as the gap in the profile of $\left|B_{\theta}^{\sim}\right|$ near $r \approx r_{\text {res }}$. The minimum value of this gap is shifted to the plasma depth from the surface $r=r_{\text {res. }}$. These results are in qualitative agreement with HYBTOK-II experimental measurements of $\left|B_{r}^{\sim}\right|$ and $\left|B_{\theta}^{\sim}\right|$ profiles. The calculated width of the resonant zone is not small: $\Delta r \sim 1 \mathrm{~cm}$.

Note, that for the CSTN-IV and HYBTOK-II calculations we took the safety factor $q(r)$ profile in the form of equation (2), from $[3,4]$.
In the experiments reported in [3-6] the radial profiles of the total magnetic perturbations were measured. In our calculations only the main resonant mode $(m / l=6 / 1)$ is investigated and the influence of the sideband modes $(\mathrm{m} / \mathrm{l}=$ $7 / 1,5 / 1)$ is not taken into account. For example, the mode $m / l=7 / 1$ may be responsible for the amplification of $\left|B_{r}^{\sim}\right|$ outside the resonant surface of the main mode.

When the phase velocity of the perturbation is almost equal to the $\boldsymbol{E} \times \boldsymbol{B}$ drift velocity (case I for CSTN-IV and case II for HYBTOK-II), equation (9) may contain a small parameter in the derivative. In this case, the radial profile of the $\boldsymbol{E} \times \boldsymbol{B}$ drift velocity must be taken into account. That means that this paper describes case II for CSTN-IV or case I for HYBTOK-II rather than case I for CSTN-IV and case II for HYBTOK-II.

These are possible reasons why some differences exist between our present calculations and experiments [3-6]. 

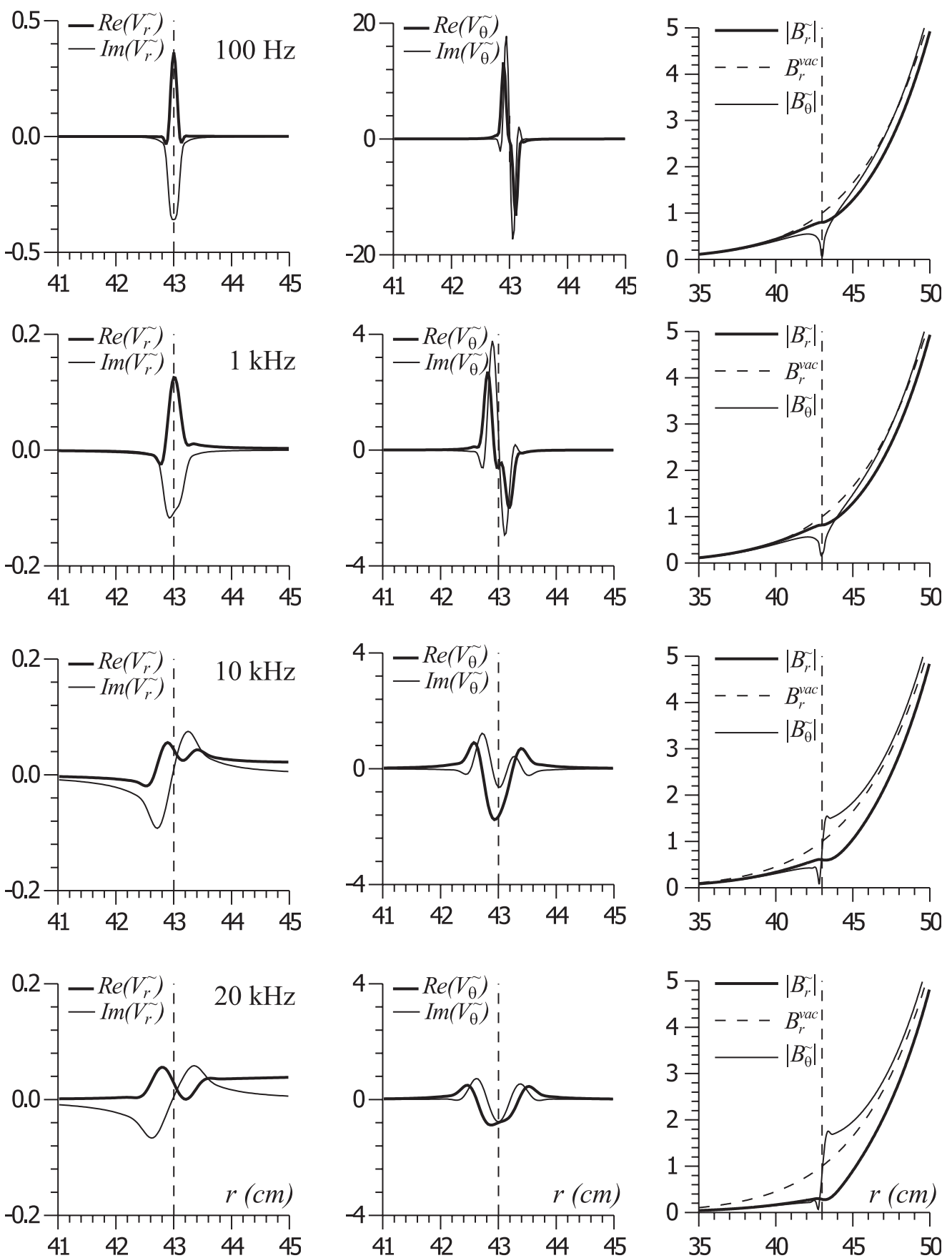

Figure 3. The TEXTOR-DED tokamak: the radial profiles of the velocities $V_{r}^{\sim}, V_{\theta}^{\sim}$ and profiles of the magnetic perturbation amplitudes $\left|B_{r}^{\sim}\right|,\left|B_{\theta}^{\sim}\right|$ for the various frequency values.

The results of the calculations depend on the local values of $Z_{\text {eff }}$. Unfortunately, the information about the local values of $Z_{\text {eff }}$ is not presented in [3-6]. The estimates show (equation (16)) that in these experiments the resistive effects are dominating in a broader region than that defined by the Alfvén resonances.

\section{TEXTOR-DED}

In this section, in figures 3 and 4 the calculations for the TEXTOR-DED tokamak are presented $(R=1.75 \mathrm{~m}$, $a=0.47 \mathrm{~m}, r_{\text {res }}=43 \mathrm{~cm}, m=12, l=4, B_{z 0}=2.25 \mathrm{~T}$, $n=10^{19} \mathrm{~m}^{-3}$ ). We considered four cases, $f^{\prime}=100 \mathrm{~Hz}$, $f^{\prime}=1 \mathrm{kHz}, f^{\prime}=10 \mathrm{kHz}$ and $f^{\prime}=20 \mathrm{kHz}$. For $f=1 \mathrm{kHz}$ $\delta=2.2 \mathrm{~cm}\left(T_{\mathrm{e}}=50 \mathrm{eV}\right)$. In the case of TEXTOR-DED the safety factor profile is taken in the form:

$$
q(r)=\frac{2 q(0)(r / a)^{2}}{1-\left(1-(r / a)^{2}\right)^{2}} .
$$

For $f^{\prime}=100 \mathrm{~Hz}$ and $f^{\prime}=1 \mathrm{kHz}$, when the plasma response is small, the results presented in figure 4 practically coincide with the same calculations of the paper [11]. In that paper the analytic solution of equations (9) and (10) was found assuming that the vacuum DED coil perturbation of the 

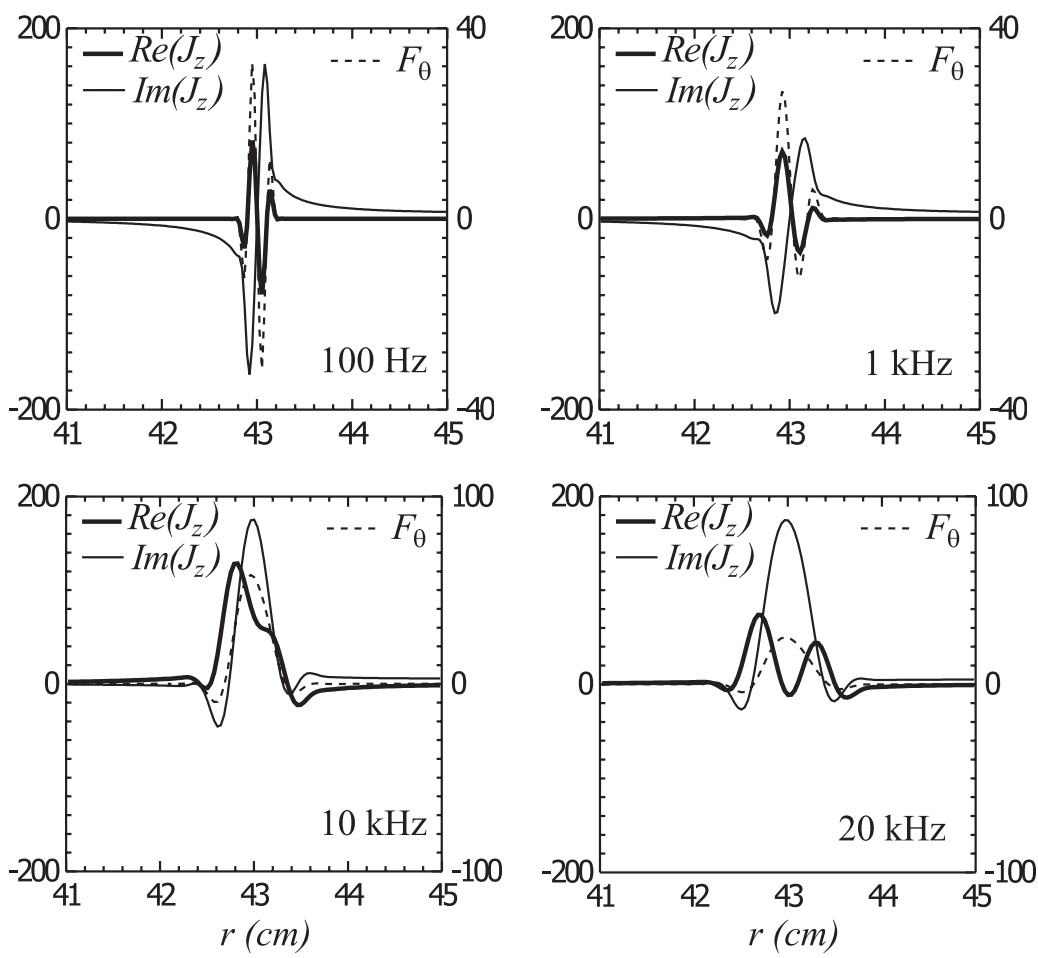

Figure 4. The TEXTOR-DED tokamak: the perturbation current density distribution $J_{z}^{\sim}$ and the radial profile of the poloidally driven force density $F_{\theta}$.

magnetic field plays a dominant role and the plasma response is small. But for $f^{\prime}=10 \mathrm{kHz}$ and $f^{\prime}=20 \mathrm{kHz}$ the plasma response is very strong and the approximations of the paper [11] are not valid.

For $f^{\prime}=100 \mathrm{~Hz}$ and $f^{\prime}=1 \mathrm{kHz}$ the width of the resonant zone is $\Delta r \sim 0.5 \mathrm{~cm}$, and for $f^{\prime}=10 \mathrm{kHz}$ and $f^{\prime}=20 \mathrm{kHz}$ $\Delta r \sim 1 \mathrm{~cm}$. Note, that the plasma response motion has a large poloidal velocity, for example, $\left|V_{\theta}^{\sim}\right| \sim(10-100) \mathrm{km} \mathrm{s}^{-1}$, if the DED coil current is the order of $5 \mathrm{kA}$. During DED experiments it is necessary to take into account the Doppler shift of the frequency: in the first DED experiments [17] the Doppler shifted frequency was very high $\left(f^{\prime} \sim 20 \mathrm{kHz}\right)$. In this case $\left|B_{r}^{\sim}\right|$ and the poloidal driven force $\left\langle F_{\theta}\right\rangle$ drops significantly.

In figure 4 the perturbation current density distribution $J_{z}^{\sim}$ and the radial profile of the poloidal driven force density $F_{\theta}$ are shown. Note that at $f^{\prime} \lesssim 1 \mathrm{kHz}$ the poloidal driven force density oscillates along the minor radius, which means that the poloidal force

$$
\left\langle F_{\theta}\right\rangle=\int F_{\theta}(r) \mathrm{d} V \propto \int_{0}^{a} F_{\theta}(r) r \mathrm{~d} r
$$

is close to zero. In figure $4, J_{z}^{\sim}$ and $F_{\theta}$ are normalized to the values $B_{r}^{\text {vac }}\left(r_{\text {res }}\right) / \mu_{0} r_{\text {res }}$ and $\left(B_{r}^{\text {vac }}\left(r_{\text {res }}\right)\right)^{2} / \mu_{0} r_{\text {res }}$, respectively.

\section{Conclusions}

The investigations of the plasma response influence on the penetration of the resonant external low frequency helical perturbation into the CSTN-IV, HYBTOK-II and TEXTOR-DED tokamak plasmas are carried out. These tokamaks have different parameters and for this reason just the same equations result in an essentially different behaviour of perturbations.
For CSTN-IV the plasma response is not so strong and the width of the interaction region is very wide, $\Delta r \sim 4 \mathrm{~cm}$; for HYBTOK-II the plasma response is stronger and the width of the interaction region is $\Delta r \sim 1 \mathrm{~cm}$. The amplification of the $B_{r, \theta}^{\sim}$ amplitudes towards the plasma depth is found for these tokamaks. A good qualitative agreement with the experiments is observed.

For TEXTOR-DED a strong response is seen for $f^{\prime} \gtrsim 5 \mathrm{kHz}$, while for $f^{\prime} \lesssim 1 \mathrm{kHz}$ the plasma response is not so strong. A narrow resonant zone, $\Delta r \sim 0.5 \mathrm{~cm}$, is observed. Note that it is much larger than the ion gyroradius. For TEXTOR-DED the poloidal magnetic field of the vacuum perturbation is practically completely compensated for by the plasma response at $r=r_{\text {res }}$. For $f^{\prime} \gtrsim 5 \mathrm{kHz}$ the poloidally driven force may produce the momentum transfer to the plasma: the poloidally driven force density oscillations along the minor radius are lacking in this case. For high frequencies $\left(f^{\prime} \sim 20 \mathrm{kHz}\right.$ ) the value $\left|B_{r}^{\sim}\right|$ and the poloidally driven force strongly decrease.

The velocity $V_{r}^{\sim}$ has a finite value in the region from the resonant zone up to the plasma boundary. For example, for $f \sim 10 \mathrm{kHz}$ the velocity near the plasma boundary $\left|V_{r}^{\sim}\right| \sim$ $500 \mathrm{~m} \mathrm{~s}^{-1}$, which means $\xi_{r} \sim V_{r}^{\sim} / \omega \sim 1 \mathrm{~cm}\left(\xi_{r}\right.$ is the radial plasma displacement), when the current in the DED coils is equal to $15 \mathrm{kA}$. An enhancement of the radial plasma flux, thus, occurs.

For the experimental conditions considered (CSTN-IV, HYBTOK-II and TEXTOR-DED) the resistive effects play a dominant role in the region that is large in comparison with the region defined by the Alfvén resonances.

In this paper, the problem was considered in the linear approximation. The estimates show that the linear model is valid for TEXTOR-DED, if DED coil current is less than or of 
the order of $5 \mathrm{kA}$. In the opposite case, the nonlinear regime may be expected; the nonlinear radial plasma flows and current may occur (see, e.g. [18]).

\section{Acknowledgments}

The authors would like to thank S. Takamura, Y. Kikuchi and M. Kobayashi for fruitful discussions. A part of this work was carried out within the framework of the WTZ project UKR-01/003 between Germany and Ukraine.

\section{References}

[1] Finken K.H., Abdullaev S.S., Kaleck A. and Wolf G.H. 1999 Nucl. Fusion 39637

[2] Boozer A.H. 1996 Phys. Plasmas 34620

[3] Kobayashi M., Tuda T., Tashiro K., Kojima H., Zhai K. and Takamura S. 2000 Nucl. Fusion 40181

[4] Kobayashi M., Kojima H., Zhai K. and Takamura S. 2000 Phys. Plasmas 73288

[5] Kikuchi Y., Kojima H., Taniyama T., Toyoda M., Uesugi Y. and Takamura S 2001 Proc. 28th EPS Conf. on Control. Fusion and Plasma Phys. (Funchal, June 2001) vol 25A (ECA) p 277
[6] Takamura S., Kikuchi Y., Uesugi Y. and Kobayashi M. 2003 Nucl. Fusion 43393

[7] Braginskii S.I. 1965 Reviews of Plasma Physics vol 1 (New York: Consultants Bureau) p 205

[8] Basu B. and Coppi B. 1977 Nucl. Fusion 171245

[9] Mikhailovskii A.B. 1998 Plasma Phys. Control. Fusion 40 1907

[10] Bateman G. 1979 MHD Instability (Cambridge, MA: MIT Press)

[11] Pankratov I.M., Omelchenko A.Ya. and Olshansky V.V. 2002 Problems of Atomic Science and Technology. Series: Plasma Physics vol 8, p 3

[12] Migliuolo S. 1993 Nucl. Fusion 331721

[13] Pankratov I.M., Omelchenko A.Ya. and Olshansky V.V. 2003 Proc. 30th EPS Conf. on Control. Fusion and Plasma Phys. (St. Petersburg, July 2003) vol 27A (ECA) P-2.137

[14] Lazzaro E., Gianoli L. and Valdettaro L. 1998 Plasma Phys. Control. Fusion $\mathbf{4 0} 1327$

[15] Faulconer D.W. and Koch R. 1997 Fusion Eng. Des. 37399

[16] Kantorovich L.V. and Akilov G.P. 1982 Functional Analysis (Oxford: Pergamon)

[17] Finken K.H. et al 2003 Proc. 30th EPS Conf. on Control. Fusion and Plasma Phys. (St Petersburg, July 2003) vol 27A (ECA) O-4.4Apd

[18] Klima R. and Peterzilka V. 1980 Czech. J. Phys. B 301002 